\title{
Palmitate-Induced Cell Death and Mitochondrial Respiratory Dysfunction in Myoblasts are Not Prevented by Mitochondria-Targeted Antioxidants
}

\author{
Jana Patková Michal Anděl Jan Trnka \\ Department of Nutrition and Centre for Research on Diabetes, Metabolism and Nutrition, Third Faculty \\ of Medicine, Charles University in Prague, Czech Republic
}

\section{Key Words}

Palmitate $\cdot$ Mitochondrial respiration $\cdot$ mtDNA integrity Mitochondria-targeted antioxidants

- MitoQ • MitoTEMPOL $\cdot$ Skeletal muscle $\cdot$ Myoblasts $\cdot$ Myotubes $\cdot$ Spare respiratory capacity

\begin{abstract}
Background/Aims: Deleterious effects of saturated fatty acids in skeletal muscle cells are well known but their impact on mitochondrial respiration has not been well studied. Mitochondrial oxidative damage has been implicated to play a role in their effect. The purpose of this study was to evaluate viability, mtDNA integrity and mitochondrial respiration in $\mathrm{C} 2 \mathrm{C} 12$ myoblasts and myotubes exposed to palmitate and to test the effect of mitochondria-targeted antioxidants MitoQ and MitoTEMPOL in preventing palmitate-induced damage. Methods: Cells were treated with tested compounds, mtDNA damage was detected by quantitative PCR and mitochondrial respiration was measured using an extracellular flux analyzer XF24. Results: Palmitate caused mtDNA damage, which was associated with reduced mitochondrial respiration and cell death in myoblasts but not in myotubes. MitoTEMPOL was able to prevent palmitate-induced mtDNA damage in myoblasts but failed to prevent cell death. MitoQ did not show any protective effect and both compounds markedly inhibited mitochondrial respiration. Conclusion: Our results indicate that skeletal muscle progenitor cells could be the first target of the deleterious action of palmitate, as myoblasts appeared to be more sensitive to its effects than myotubes possibly in part due to a lower spare respiratory capacity in the former. Only MitoTEMPOL prevented palmitate-induced mtDNA damage but neither antioxidant was able to prevent cell death and both antioxidants had a marked negative effect on respiration.
\end{abstract}




\section{Introduction}

Elevated levels of plasma free fatty acids (FFA), which are characteristic for obesity and type 2 diabetes [1, 2], are associated with various pathophysiological consequences in skeletal muscle, although recently this simple association has been put into question [3]. Development of insulin resistance and changes in mitochondrial function in skeletal muscle have been found in healthy humans and animals after lipid infusion [4, 5] or after prolonged fasting [6]. Experiments on cultured skeletal muscle cells show that exposure to longchain saturated FFA but not unsaturated FFA leads to the induction of insulin resistance, inflammation, some features of mitochondrial dysfunction and apoptosis [7-9]. Although information has accumulated regarding the role of FFA in the induction of insulin resistance in skeletal muscle and cultured skeletal muscle cells $[4,5,7,10]$ less data is available about their effect on mitochondrial function and specifically mitochondrial respiration.

Metabolic states characterized by hyperglycemia and high levels of circulating free fatty acids have been shown to be associated with a chronic oxidative stress and increased mitochondrial reactive oxygen species (ROS) production [11, 12]. Increased production of hydrogen peroxide associated with insulin resistance was observed also in rodents on high fat diet with no change in mitochondrial respiratory function [13]. There are several studies in cultured skeletal muscle cells showing an increase of mitochondrial ROS production induced by palmitate [14-16] accompanied by mtDNA damage and some features of mitochondrial dysfunction $[17,18]$. Mitochondrial DNA has an important role in the regulation of mitochondrial respiration and oxidative damage of mtDNA was postulated as an initial event leading to mitochondrial dysfunction [18].

Therapy that can decrease mitochondrial oxidative damage could prevent damage associated with excess of nutrients such as saturated fatty acids. Mitochondria-targeted antioxidants accumulate in mitochondria and have been shown to be more effective in preventing mitochondrial oxidative damage than untargeted antioxidants [19,20]. MitoQ and MitoTEMPOL are two promising compounds, whose targeting to mitochondria is achieved by the covalent attachment of an antioxidant molecule to the lipophilic triphenylphosphonium cation $\left(\mathrm{TPP}^{+}\right)$[21]. The shielded positive charge of these lipophilic cations enables them to permeate lipid bilayers easily and to accumulate several hundred-fold within mitochondria, due to the large membrane potential [22]. MitoQ is a ubiquinone derivative which exhibited both antioxidant and anti-apoptotic properties in a large number of in vitro as well as in vivo studies [19, 23-26]. MitoTEMPOL consists of the piperidine nitroxide TEMPOL conjugated with a $\mathrm{TPP}^{+}$moiety. It has been shown to be effective in preventing lipid peroxidation and mtDNA damage in skeletal muscle cells [27] or in protecting pancreatic $\beta$-cells against oxidative stress under glucolipotoxic conditions [24].

The purpose of the present study was to clarify the effect of palmitate on viability, mtDNA integrity and mitochondrial respiration in C2C12 skeletal muscle cells. We tested the effect of palmitate both on differentiated myotubes and undifferentiated myoblasts. We found palmitate-induced mtDNA damage which was associated with reduced mitochondrial respiration, increased production of hydrogen peroxide and cell death in myoblasts, but not in myotubes. Following on the hypothesis that palmitate caused damage through an induction of oxidative stress, we hypothesized that treatment with mitochondria-targeted antioxidants might prevent this damage in myoblasts and we tested two such antioxidants, MitoQ and MitoTEMPOL.

\section{Materials and Methods}

\section{Materials}

Mitochondria-targeted antioxidants MitoQ and MitoTEMPOL were kindly provided by Dr. Michael P. Murphy (MRC Mitochondrial Biology Unit, Cambridge, UK). Propyltriphenylphosphonium bromide, palmitate and bovine serum albumin (fatty acid free) were purchased from Sigma-Aldrich. Fetal bovine 
serum for cell cultures was from Life Technologies, penicillin and streptomycin were from Sigma-Aldrich. Blood \& Cell Culture DNA Mini Kit for DNA isolation was obtained from Qiagen and reagents for QPCR from Applied Biosystems.

\section{Cell culture and treatment}

As a model of skeletal muscle we used murine C2C12 myoblasts. Cells were obtained from the European Collection of Cell Cultures and were used as myoblasts, which served as a model of satellite cells, and as myotubes, an in vitro model of muscle fibres. Cells were grown in Dulbecco's modified Eagle's medium (DMEM, Life Technologies) supplemented with 10\% fetal bovine serum, $100 \mathrm{U} / \mathrm{ml}$ penicillin and $100 \mu \mathrm{g} / \mathrm{ml}$ streptomycin at $37^{\circ} \mathrm{C}$ in an atmosphere of $95 \%$ humidity and $5 \% \mathrm{CO}_{2}$ and maintained below a maximal confluence of $70-80 \%$. To initiate differentiation, cells were allowed to reach $100 \%$ confluence and the medium was changed to the differentiation medium (DMEM containing $2 \%$ fetal bovine serum), which was replaced every $48 \mathrm{~h}$. Myotubes were used for experiments after 7 days, when full differentiation was observed.

A stock concentration of palmitate was prepared by conjugating palmitate with fatty acid-free bovine serum albumin (BSA), using a modified method described by Cousin [28]. Briefly, palmitate was dissolved in $0.1 \mathrm{M}$ sodium hydroxide at $70^{\circ} \mathrm{C}$ to a final concentration of $100 \mathrm{mM}$. Palmitate was then mixed with $15 \%$ fatty acid-free BSA at $50^{\circ} \mathrm{C}$ for $30 \mathrm{~min}$, yielding a final stock solution of $5 \mathrm{mM}$. The molar ratio of FFA/BSA was 2:1 in this preparation which is close to the ratio observed in human serum [29]. A control BSA solution was prepared by mixing a volume of $0.1 \mathrm{M}$ sodium hydroxide equal to that of palmitate in the palmitate-BSA solution with $15 \%$ fatty acid-free BSA. The $\mathrm{pH}$ of both solutions was adjusted to 7.4. After sterilization by filtration the actual concentration of palmitate was determined using the NEFA (non-esterified fatty acids) kit (Randox Laboratories, UK).

Cells were incubated for $18 \mathrm{~h}$ in DMEM containing palmitate-BSA conjugate or DMEM with BSA only (control). For experiments with antioxidants MitoQ or MitoTEMPOL were added to cells $1 \mathrm{~h}$ prior to the addition of palmitate and then incubated for $18 \mathrm{~h}$. All compounds were washed away before measurements. We also treated cells with corresponding concentrations of propylTPP (рTPP) to control for the effect of the cation moiety itself [30].

\section{Cell viability assay}

Cell viability after the exposure to various concentrations of palmitate and antioxidants was assessed using the MTS assay (Promega). The incubation medium with tested compounds was changed to a medium with the MTS reagent at the end of exposure and cells were incubated for another $2 \mathrm{~h}$ at $37^{\circ} \mathrm{C}$. The absorbance of dissolved formazan was measured at $490 \mathrm{~nm}$ in a microplate reader. Data are displayed as a percentage of cells with no additions.

\section{Detection of extracellular hydrogen peroxide}

Extracellular hydrogen peroxide $\left(\mathrm{H}_{2} \mathrm{O}_{2}\right)$ was measured using the Amplex Red reagent (Life Technologies) by monitoring its oxidation to fluorescent resorufin in the presence of horseradish peroxidase (HRP). Cells were grown in 96-well plates and treated as described above. The incubation medium was then removed and cells were incubated with the reaction mixture at $37^{\circ} \mathrm{C}$ for $30 \mathrm{~min}$ protected from light. The reaction mixture consisted of $50 \mu \mathrm{M}$ Amplex Red reagent and $0.1 \mathrm{U} / \mathrm{ml}$ HRP in Krebs-Ringer phosphate $(145 \mathrm{mM}$ $\mathrm{NaCl}, 5.7 \mathrm{mM}$ sodium phosphate, $4.86 \mathrm{mM} \mathrm{KCl}, 0.54 \mathrm{mM} \mathrm{CaCl}, 1.22 \mathrm{mM} \mathrm{MgSO}_{4}, 5.5 \mathrm{mM}$ glucose, $\mathrm{pH}$ 7.35). Fluorescence was detected at $590 \mathrm{~nm}$ with excitation at $540 \mathrm{~nm}$. Protein content in each well was then determined by the bicinchoninic acid kit (Sigma). Data are expressed as relative fluorescence units (RFU) of resorufin $\left(\mathrm{H}_{2} \mathrm{O}_{2}\right.$ emission) per microgram of protein.

\section{Analysis of mtDNA damage by quantitative PCR}

The integrity of mtDNA was assessed following treatments, using the quantitative polymerase chain reaction (QPCR) methodology [31,32] with some modifications. The QPCR assay is based on the fact that any damage to DNA blocks the progression of the DNA polymerase on a template and will result in a decreased amplification. Amplification is thus inversely proportional to the amount of DNA damage on a given template. 
Cells were incubated with tested compounds in $6 \mathrm{~cm}$ Petri dishes and high molecular weight DNA was then isolated with the Blood \& Cell Culture DNA Mini Kit (Qiagen) as described in the manufacturer's protocol with some modifications. The tissue protocol was used, since the protocol for DNA extraction of cultured cells involves an isolation of nuclei and hence a loss of mtDNA [31]. The concentration of total cellular DNA was determined fluorometrically using Qubit dsDNA HS Assay Kit and Qubit fluorometer (Life Technologies). Stock DNA was diluted in TE buffer (10 mM Tris-HCl, 1 mM EDTA, pH 8.0) to a final concentration of $3 \mu \mathrm{g} / \mathrm{ml}$, which was used as a template for PCR. Two targets of mtDNA were amplified: a long target to quantify levels of mtDNA lesions and a short target to control for changes in mtDNA copy number. The long target $(\approx 10 \mathrm{kbp})$ spanned ND1 and ND5 genes and the small target (127 bp) was part of ND1 gene. The primers were synthesized by Sigma and the primer nucleotide sequences were as follows: 5'-GCC AGC CTG ACC CAT AGC CAT AAT-3' and 5'-GCC GGC TGC GTA TTC TAC GTT A-3' for short target, 5'-GCC AGC CTG ACC CAT AGC CAT AAT-3' and 5'-GAG AGA TTT TAT GGG TGT AAT GCG G-3' for long target. The total volume for each PCR reaction was $20 \mu \mathrm{l}$, consisting of $15 \mathrm{ng}$ of DNA template, $1 \mathrm{U}$ rTth DNA polymerase XL (Applied Biosystems), $1 \times$ buffer XL (provided with the rTth polymerase), $200 \mu \mathrm{M}$ dNTPs, 20 pmol forward primer, $20 \mathrm{pmol}$ reverse primer, $100 \mathrm{\mu g} / \mathrm{ml}$ of $\mathrm{BSA}, 1 \mathrm{mM} \mathrm{Mg}(\mathrm{OAc})_{2}$ for long target and $1.5 \mathrm{mM} \mathrm{Mg}(\mathrm{OAc})_{2}$ for short target. To ensure quantitative conditions, a control reaction containing $50 \%$ of template DNA was included during each set of amplification and only amplification ranging from 40 to $60 \%$ was considered acceptable. Automated hot-start using AmpliWax PCR Gems 50 (Applied Biosystems) was used to increase sensitivity and specificity of PCR. The parameters for the short mitochondrial target were $1 \mathrm{~min}$ at $94^{\circ} \mathrm{C}$, then 20 cycles of $30 \mathrm{~s}$ at $94^{\circ} \mathrm{C}, 45 \mathrm{~s}$ at $64^{\circ} \mathrm{C}, 45 \mathrm{~s}$ at $72^{\circ} \mathrm{C}$, followed by a final extension step of $10 \mathrm{~min}$ at $72^{\circ} \mathrm{C}$. The parameters for the long mitochondrial target were $1 \mathrm{~min}$ at $94^{\circ} \mathrm{C}$, then 18 cycles of $15 \mathrm{~s}$ at $94^{\circ} \mathrm{C}, 12$ $\min$ at $64^{\circ} \mathrm{C}$, followed by a final extension step of $10 \mathrm{~min}$ at $72^{\circ} \mathrm{C}$. The PCR products were then quantified fluorometrically and the results were expressed as amplification relative to the control, which was defined as undamaged for the purposes of this assay.

\section{Analysis of mitochondrial respiration}

Oxygen consumption rates (OCR) and extracellular acidification rates (ECAR) were measured using an extracellular flux analyzer XF24 (Seahorse Bioscience). The assay medium consisted of modified DMEM lacking sodium bicarbonate (XF Assay medium, Seahorse Bioscience) supplemented with $4 \mathrm{mM} \mathrm{L-glutamine,}$ $1 \mathrm{mM}$ pyruvate and $5.5 \mathrm{mM}$ glucose. The $\mathrm{pH}$ was adjusted to 7.4 at $37^{\circ} \mathrm{C}$ on the day of the experiment.

For experiments with myoblasts cells were seeded at a density of 13,000 cells per well in 24-well plates designed for this instrument and left to attach for at least $2 \mathrm{~h}$. Cells were then treated with tested compounds as described above. For experiments with myotubes cells were differentiated for 7 days in assay plates prior to treatment. The culture medium was replaced with the assay medium $1 \mathrm{~h}$ prior to measurement and cells were kept at $37^{\circ} \mathrm{C}$ in an atmosphere without $\mathrm{CO}_{2}$. Mitochondrial stress test was then performed to assess the bioenergetic status of the cells [33]. OCR measurements were obtained before and after sequential additions of the ATPase inhibitor oligomycin $(1 \mu \mathrm{M})$, inner membrane uncoupler carbonyl cyanide 4-(trifluoromethoxy)phenylhydrazone (FCCP, $1 \mu \mathrm{M}$ ) and inhibitors of complex I and III rotenone $(1 \mu \mathrm{M})$ and antimycin $\mathrm{A}(1 \mu \mathrm{M})$ in myoblasts. For myotubes the concentration of FCCP was increased to $1.5 \mu \mathrm{M}$ to obtain a maximal effect. After completion of the measurement, assay medium was removed and well contents were lysed in CelLytic MT lysis buffer (Sigma). Protein content in each well was determined by bicinchoninic acid kit (Sigma). The following parameters were then calculated: basal respiration (baseline respiration before addition of compounds), ATP turnover-linked respiration (oligomycin-sensitive respiration), maximal respiration (maximal uncoupled respiration), spare respiratory capacity (maximal uncoupled respiration minus basal respiration) and proton leak (oligomycin-insensitive respiration). Basal respiration is controlled strongly by ATP turnover, which alters mainly in response to ATP demand in the cell, and by changes in proton leak [34]. Maximal respiration after the addition of an uncoupler then reflects the maximal capacity of the electron transport chain. Non-mitochondrial respiration (after the addition of rotenone/antimycin A) was subtracted from basal and maximal respiration and proton leak. Data are expressed as pmol $\mathrm{O}_{2} / \mathrm{min} / \mu \mathrm{g}$ protein. Coupling efficiency (CE) was calculated as the ratio of ATP turnoverlinked respiration and basal respiration [33].

Glycolytic activity (ECAR) was measured in parallel with respiration and the following parameters were calculated: basal ECAR (before addition of compounds), oligomycin-stimulated ECAR, and glycolytic reserve capacity (oligomycin-stimulated minus basal ECAR). Data are expressed as $\mathrm{mpH} / \mathrm{min} / \mu \mathrm{g}$ protein. 
Fig. 1. Palmitate decreased viability of myoblasts (A) and myotubes (B) in a dose-dependent manner. Cells were treated with palmitate (PA) in concentrations varying from 0.01 to $1 \mathrm{mM}$ or corresponding concentrations of BSA (C) for $18 \mathrm{~h}$ and cell viability was assessed using MTS assay. The effect of palmitate was statistically significant from $0.05 \mathrm{mM}$ PA in myoblasts and $0.3 \mathrm{mM}$ PA in myotubes. Only the highest concentration of BSA (corresponding to 1 $\mathrm{mM}$ palmitate) significantly decreased viability of myoblasts. Data are displayed as a percentage of untreated cells and presented as means and 95\% CI from five independent experiments.

\section{Citrate synthase activity measurement}

The citrate synthase activity was determined in whole cell extracts using the Citrate Synthase Assay Kit (Sigma). The assay mixture contained $0.3 \mathrm{mM}$ acetyl coenzyme A, $0.5 \mathrm{mM}$ oxaloacetate and $0.1 \mathrm{mM}$ 5,5-dithiobis(2-nitrobenzoic acid). Protein content of samples was determined by the bicinchoninic acid kit. The enzyme activity was measured spectrophotometrically at $30^{\circ} \mathrm{C}$ at $412 \mathrm{~nm}$ and expressed in $\mathrm{nmol} / \mathrm{min} / \mathrm{mg}$ protein.

\section{Statistical analysis}

All data represent at least three experiments and are expressed as means and 95\% confidence intervals (CI, in square brackets). Mixed-effects model ANOVA with treatment as the fixed effect and set of experiment as the random effect was used followed by Dunnett post-hoc test for multiple comparisons. All analyses were performed in Statistica 9.1 (StatSoft, Inc.). P values less then 0.05 were considered statistically significant.
A

B
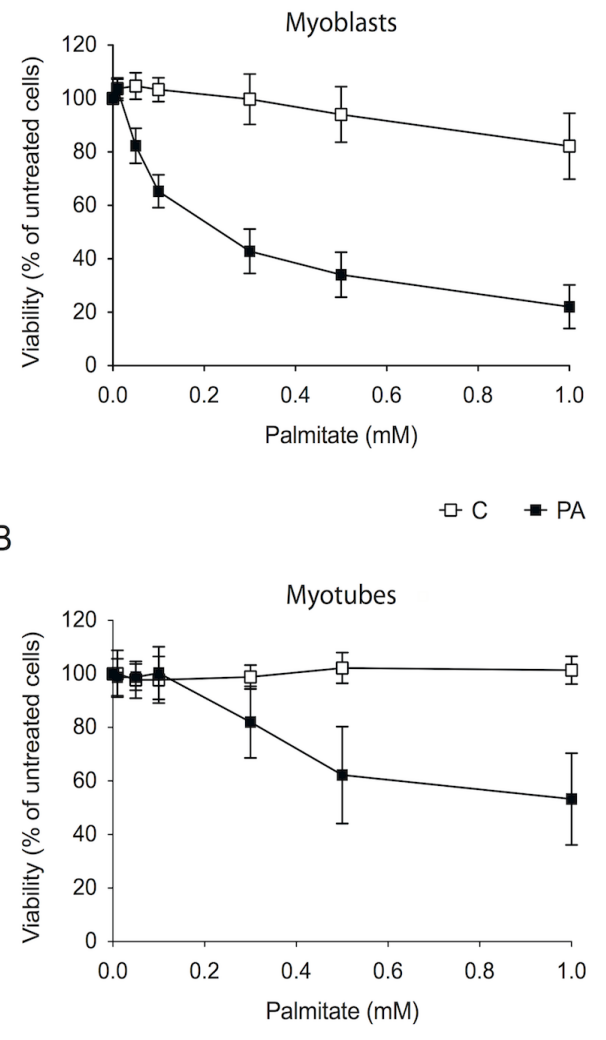

\section{Results}

Palmitate decreased cell viability and caused mtDNA damage in myoblasts and myotubes

We evaluated the cytotoxic effect of palmitate on myoblasts and myotubes in the concentration range of $0.01-1 \mathrm{mM}$. Cells were exposed to palmitate-BSA conjugate or to corresponding concentrations of BSA only. The results of MTS assays show a dose-dependent reduction of viability in both myoblasts and myotubes after an $18 \mathrm{~h}$ exposure to palmitate (Fig.1A and B). Myoblasts showed a higher sensitivity to the cytotoxic effect of palmitate than differentiated myotubes. Only the highest concentration of BSA (corresponding to 1 $\mathrm{mM}$ palmitate) led to significantly decreased viability of myoblasts (Fig. 1A).

Based on toxicity assays $100 \mu \mathrm{M}$ concentration of palmitate was chosen and its effect on mtDNA integrity was tested. We consider this concentration of palmitate as physiologically relevant: although total plasma fatty acids can be as high as $1 \mathrm{mM}$ or even higher under some physiological and pathological conditions (prolonged fasting, obesity, type 2 diabetes) [4], the concentration of palmitate under most conditions is generally much lower [35]. We used the QPCR assay to monitor damage to mtDNA by comparing the relative amplification of a very long fragment of mtDNA from treated and non-treated samples. As shown in Fig. $2 \mathrm{~A}$ the relative amplification of the long mtDNA fragment was decreased both in myoblasts (difference of means C vs. PA -15.18 [-27.78, -2.59]) and myotubes (-16.84 [-29.68, -4.01]) after palmitate exposure suggesting that palmitate caused similar mtDNA damage. 
A

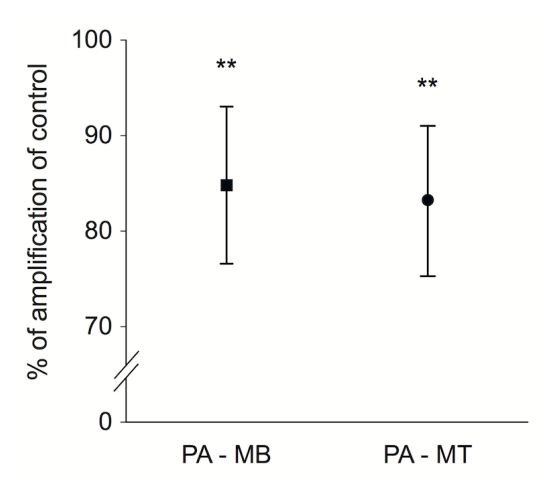

B

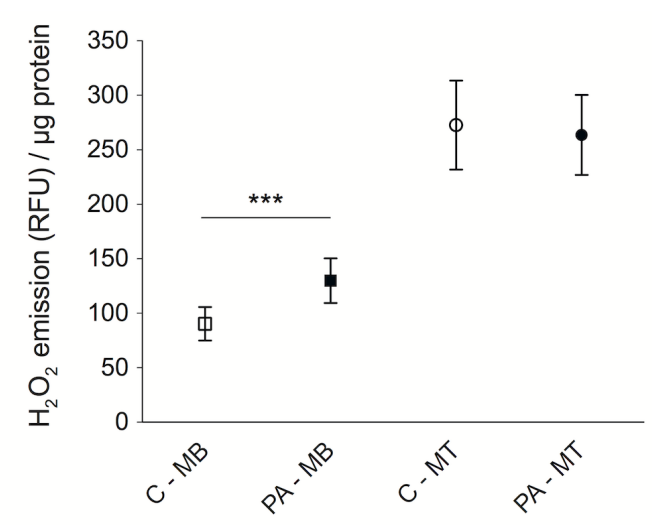

Fig. 2. Palmitate-induced mtDNA damage in myoblasts (PA-MB) and myotubes (PA-MT) and increased $\mathrm{H}_{2} \mathrm{O}_{2}$ production in myoblasts. Cells were treated with $100 \mu \mathrm{M}$ palmitate or $0.3 \%$ BSA for $18 \mathrm{~h}$. A) integrity of mtDNA was assessed by QPCR, where mtDNA of controls was defined as undamaged (100\% amplification). Palmitate significantly decreased relative amplification of $10 \mathrm{~kb}$ fragment of mtDNA both in myoblasts and myotubes. B) extracellular $\mathrm{H}_{2} \mathrm{O}_{2}$ was measured by monitoring Amplex Red oxidation to fluorescent resorufin. Palmitate significantly increased $\mathrm{H}_{2} \mathrm{O}_{2}$ production only in myoblasts. Results are presented as means and $95 \% \mathrm{CI}$ from at least four independent experiments. ${ }^{* *} \mathrm{p}<0.01,{ }^{* * *} \mathrm{p}<0.001$ compared to controls.

Palmitate increased reactive oxygen species (ROS) production in myoblasts but not in myotubes

Extracellular hydrogen peroxide was measured to evaluate the role of ROS in the effect of palmitate. As shown in Fig. 2B myoblasts exposed to $100 \mu \mathrm{M}$ palmitate showed significantly higher hydrogen peroxide production compared to controls (difference of means 39.54 $[15.10,63.97] \mathrm{RFU} / \mu \mathrm{g}$ protein). Myotubes showed higher basal levels of hydrogen peroxide in controls, which were not further increased after the treatment with palmitate $(-9.03$ $[-60.73,42.66])$.

\section{Palmitate affected mitochondrial respiration more in myoblasts than in myotubes}

After treatment with $100 \mu \mathrm{M}$ palmitate for $18 \mathrm{~h}$, the bioenergetic status of cells was assessed as shown in Fig. 3A. Palmitate exposure led to a changed bioenergetic profile of both myoblasts and myotubes but in a different manner, as shown in Fig. 3B and C. In myoblasts palmitate exposure led to a decreased basal respiration (difference of means $-2.88[-5.98,0.22] \mathrm{pmol} \mathrm{O}_{2} / \mathrm{min} / \mu \mathrm{g}$ protein), ATP turnover-linked respiration (-2.86 [-5.47, $-0.25])$, maximal respiration $(-10.59[-17.68,-3.50])$ and spare respiratory capacity (SRC) $(-7.71[-11.94,-3.47])$ compared to controls while there was virtually no difference in proton leak (-0.02 [-0.58, 0.53], Fig. 3B).

In myotubes exposed to palmitate basal respiration was significantly increased compared to controls (difference of means $1.16[0.15,2.16]$ ) due to a higher proton leak $(0.60[0.31,0.90])$ but other parameters were not significantly affected (difference of means for ATP-turnover linked respiration $0.55[-0.22,1.32]$, maximal respiration 2.44 [-2.74, 7.62] and SRC 1.29 [-3.25, 5.82], Fig. 3C). We found a decrease in coupling efficiency (CE) in both myoblasts (difference of means C vs. PA $-0.04[-0.06,-0.02])$ and myotubes $(-0.05$ $[-0.06,-0.03])$ exposed to palmitate (Fig. 3D). There was no difference in non-mitochondrial respiration between palmitate-treated and control cells in either group (data not shown). Extracellular acidification rates did not differ significantly between palmitate-treated and control myoblasts or myotubes suggesting no difference in glycolytic activity (Table 1).

Citrate synthase activity was decreased in palmitate-treated myoblasts but not in myotubes

Citrate synthase (CS) activity was measured as a mitochondrial mass marker. CS activity decreased significantly in myoblasts after the treatment with palmitate (difference of means 
A

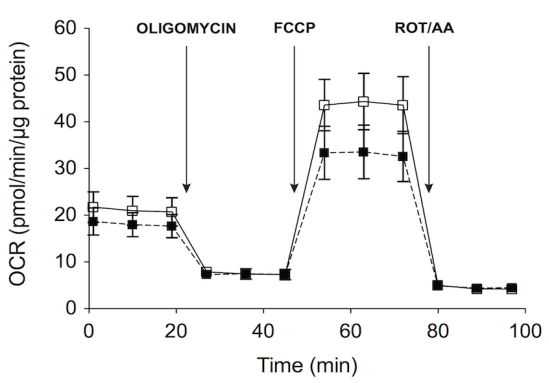

C

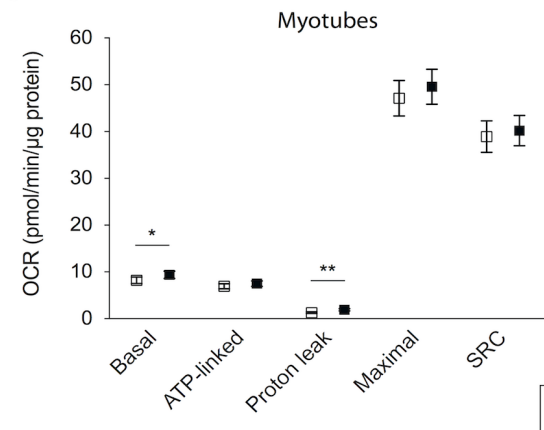

B

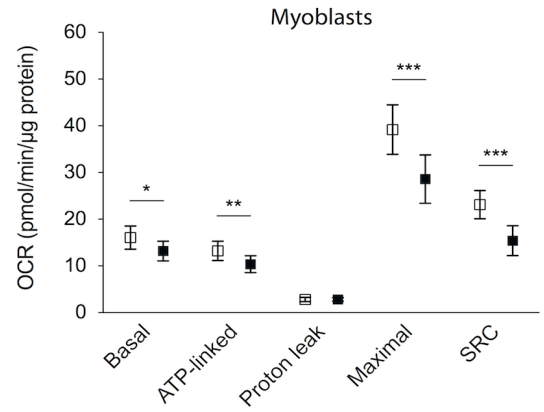

D

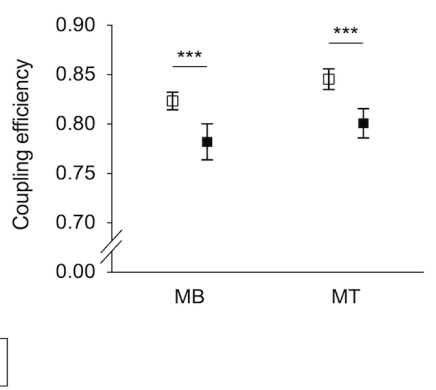

Fig. 3. Effect of palmitate exposure on mitochondrial respiration (oxygen consumption rates, OCR) in myoblasts and myotubes measured under different respiratory conditions. Cells were treated with $100 \mu \mathrm{M}$ palmitate (PA) or $0.3 \%$ BSA (C) for $18 \mathrm{~h}$ and then the growth media with palmitate was removed and replaced with assay media $1 \mathrm{~h}$ prior the assessment of mitochondrial function. OCR was measured before and after sequential injections of the indicated compounds. A) mitochondrial stress test experiment showing times of mitochondrial inhibitors addition to myoblasts. B,C) individual mitochondrial respiratory parameters (basal, ATP turnover-linked and maximal respiration; proton leak and spare respiratory capacity - SRC) calculated for myoblasts (B) and myotubes (C) as described in methods section. D) palmitate-induced decrease in coupling efficiency both in myoblasts (MB) and myotubes (MT). Results are presented as means and $95 \% \mathrm{CI}$ from four independent experiments. ${ }^{*} \mathrm{p}<0.05,{ }^{* *} \mathrm{p}<0.01,{ }^{* * *} \mathrm{p}<0.001$ compared to controls.

Table 1. Glycolytic activity (ECAR) in myoblasts and myotubes after the treatment with palmitate. Data are expressed as $\mathrm{mpH} / \mathrm{min} / \mu \mathrm{g}$ protein and results are presented as means and 95\% CI from four independent experiments

\begin{tabular}{lccc}
\hline & \multicolumn{1}{c}{ Basal } & Oligomycin-stimulated & Glycolytic reserve capacity \\
\hline Myoblasts & & & \\
Control & $1.83[1.44,2.23]$ & $2.85[2.26,3.44]]$ & $1.02[0.78,1.25]$ \\
PA & $2.04[1.77,2.32]$ & $3.16[2.69,3.62]$ & $1.11[0.90,1.33]$ \\
Myotubes & & & \\
Control & $0.38[0.29,0.48]$ & $0.73[0.60,0.87]$ & $0.35[0.29,0.41]$ \\
PA & $0.43[0.30,0.55]$ & $0.74[0.56,0.92]$ & $0.32[0.24,0.39]$ \\
\hline
\end{tabular}

C vs. PA -15.63 [-29.97, -1.30] $\mathrm{nmol} / \mathrm{min} / \mathrm{mg}$ protein), whereas no significant difference was observed between palmitate-treated and control myotubes $(-1.44[-39.45,36.57]$, Table 2).

Since the effects of palmitate on respiratory and mitochondrial parameters in myotubes were negligible further experiments using mitochondria-targeted antioxidants were performed only in myoblasts. 
Table 2. Citrate synthase activity in myoblasts and myotubes after the treatment with palmitate. Data are expressed as $\mathrm{nmol} / \mathrm{min} / \mathrm{mg}$ protein and results are presented as means and 95\% CI from four independent experiments. ${ }^{*} \mathrm{p}<0.05$ compared to controls

\begin{tabular}{lcc}
\hline & Control & PA \\
\hline Myoblasts & $147.36[135.86,158.87]$ & $131.73[122.08,141.37]{ }^{*}$ \\
Myotubes & $283.77[255.66,311.88]$ & $282.33[253.40,311.26]$ \\
\hline
\end{tabular}

Fig. 4. Neither MitoQ nor MitoTEMPOL prevented palmitate-induced cell death in myoblasts. Cells were pre-treated with MitoQ or MitoTEMPOL at the indicated concentrations for $1 \mathrm{~h}$ and then coincubated with palmitate for $18 \mathrm{~h}$. Cell viability was then assessed using MTS assay. Data are displayed as a percentage of untreated cells. Results are presented as means and $95 \% \mathrm{CI}$ from four independent experiments, n.s. - not significantly different from palmitate.
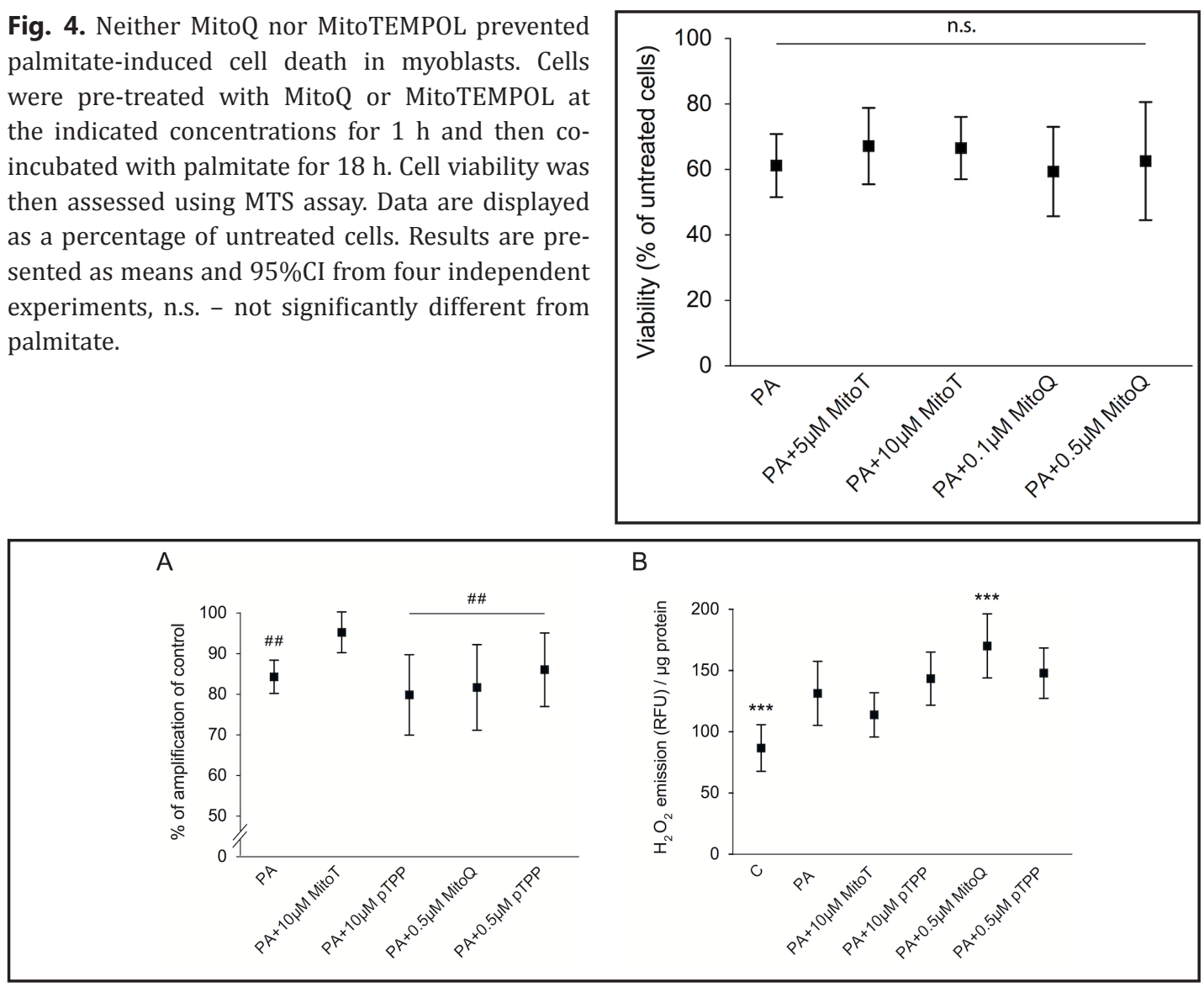

Fig. 5. MitoTEMPOL but not MitoQ decreased palmitate-induced mtDNA damage and hydrogen peroxide production in myoblasts. Cells were pre-treated with $0.5 \mu \mathrm{M}$ MitoQ $10 \mu \mathrm{M}$ MitoTEMPOL or corresponding concentrations of pTPP for $1 \mathrm{~h}$ and then co-incubated with palmitate for $18 \mathrm{~h}$. A) integrity of mtDNA was assessed by QPCR. MitoTEMPOL prevented a palmitate-induced decrease in the relative amplification of $10 \mathrm{~kb}$ fragment of mtDNA. B) extracellular $\mathrm{H}_{2} \mathrm{O}_{2}$ was measured by monitoring Amplex Red oxidation to fluorescent resorufin. MitoTEMPOL decreased palmitate-induced $\mathrm{H}_{2} \mathrm{O}_{2}$ production but the difference did not reach statistical significance. Mito $Q$ treatment led to a significantly increased $\mathrm{H}_{2} \mathrm{O}_{2}$ production. Results are presented as means and 95\%CI from three (A) or four (B) independent experiments. ${ }^{\#} \mathrm{p}<0.01$ compared to controls, *** $\mathrm{p}<0.001$ compared to palmitate.

\section{Mitochondria-targeted antioxidants had no effect on palmitate cytotoxicity}

If antioxidants are to be used in cells it is important to ensure that doses used are not toxic to a given cell type. We tested the cytotoxicity of both antioxidants in myoblasts in a broad concentration range. MitoQ significantly decreased cell viability at concentrations over $0.5 \mu \mathrm{M}$ and MitoTEMPOL for concentrations over $10 \mu \mathrm{M}$ (data not shown). Based on these results we pre-treated cells with 0.1 or $0.5 \mu \mathrm{M}$ MitoQ, 5 or $10 \mu \mathrm{M}$ MitoTEMPOL or 
Fig. 6. Effect of MitoQ MitoTEMPOL and pTPP compounds on palmitate-affected mitochondrial respiration of myoblasts. Cells were pre-treated with 0.5 $\mu \mathrm{M}$ MitoQ, $10 \mu \mathrm{M}$ MitoTEMPOL or corresponding concentrations of pTPP for $1 \mathrm{~h}$ and then co-incubated with palmitate for $18 \mathrm{~h}$. The growth medium with tested compounds was removed and replaced with the assay medium $1 \mathrm{~h}$ prior the assessment of mitochondrial function. Oxygen consumption rates (OCR) were measured before and after sequential injections of the indicated compounds. Both MitoQ and MitoTEMPOL markedly inhibited mitochondrial respiration of myoblasts compared to controls or cells exposed only to palmitate. The effect was observed also for higher concentration of pTPP $(10$ $\mu \mathrm{M})$. Results are presented as means and $95 \% \mathrm{CI}$ from three independent experiments.

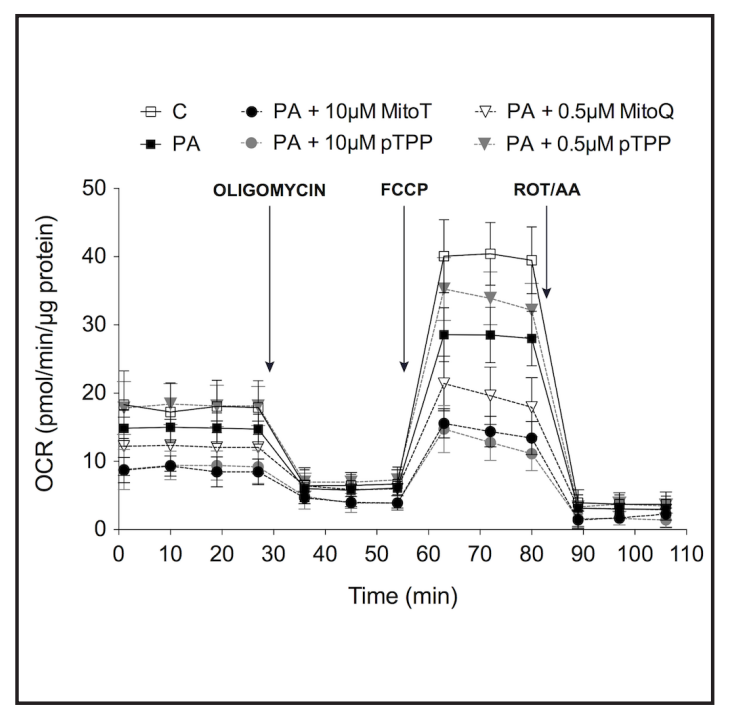

corresponding concentrations of propylTPP (pTPP) for $1 \mathrm{~h}$ and then co-incubated with palmitate for $18 \mathrm{~h}$. We found no difference in the viability of cells treated only with palmitate and cells pre-treated with two different concentrations of MitoQ, MitoTEMPOL or pTPP (Fig. 4, data for pTPP not shown). Mitochondria-targeted antioxidants MitoQ and MitoTEMPOL were therefore unable to prevent palmitate-induced cell death.

MitoTEMPOL but not MitoQ prevented palmitate-induced mtDNA damage and both antioxidants inhibited mitochondrial respiration

Based on toxicity assays and the literature $[23,27,36,37]$ we used $0.5 \mu \mathrm{M}$ concentration of MitoQ and $10 \mu \mathrm{M}$ concentration of MitoTEMPOL for subsequent experiments testing their ability to prevent palmitate-induced mtDNA damage and mitochondrial respiratory dysfunction. Treatment with MitoTEMPOL prevented palmitate-induced mtDNA damage as shown by the relative amplification of the long mtDNA fragment (difference of means $\mathrm{C}$ vs. PA -15.67 [-28.24, -3.11], C vs. PA + $10 \mu \mathrm{M}$ MitoTEMPOL -4.72 [-17.29, 7.84], Fig. 5A). No effect was observed for MitoQ and for either concentration of pTPP (difference of means $C$ vs. PA + 0.5 $\mu \mathrm{M}$ MitoQ -18.31 [-31.27, -5.36], C vs. PA + $10 \mu \mathrm{M}$ pTPP -20.14 [-32.71, -7.57], C vs. PA + $0.5 \mu \mathrm{M}$ pTPP -13.93 [-26.50, -1.37], Fig. 5A).

Both antioxidants had a profound negative effect on mitochondrial respiration (Fig. 6). They markedly inhibited basal respiration, ATP turnover-linked respiration, maximal respiration and spare respiratory capacity compared to controls or cells exposed only to palmitate. Only proton leak and non-mitochondrial respiration were not affected. Inhibition of respiration was not exclusive for antioxidants themselves but was observed also for the 10 $\mu \mathrm{M}$ concentration of pTPP pointing to a deleterious effect of the cations themselves.

The effect of Mito $Q$ and MitoTEMPOL on reactive oxygen species production in palmitatetreated myoblasts

MitoTEMPOL had a promising antioxidant potential as it significantly decreased hydrogen peroxide production in non-treated control myoblasts (difference of means $C$ vs. $10 \mu \mathrm{M}$ MitoTEMPOL -22.1 [-41.54, -2.647] RFU/ $\mu$ g protein, data not shown). In myoblasts exposed to palmitate MitoTEMPOL decreased palmitate-induced production of hydrogen peroxide but the difference did not reach statistical significance (C vs. PA 44.62 [8.13, 81.11], PA vs. PA $+10 \mu \mathrm{M}$ MitoTEMPOL -17.52 [-54.03, 18.99], Fig. 5B). MitoQ did not prevent palmitate-induced production of hydrogen peroxide and caused even increased production of this ROS (PA vs. PA + 0.5 $\mu$ M MitoQ 38.76 [2.23, 75.25], Fig. 5B). This increase was observed 
also in non-treated control cells exposed to MitoQ (C vs. 0.5 $\mu$ M MitoQ 38.97 [15.12, 62.82], data not shown). Control pTPP compounds only slightly increased the production of ROS in palmitate-treated myoblasts (not statistically significant, PA vs. PA + $10 \mu \mathrm{M}$ pTPP 12.08 [-24.41, 48.57], PA vs. PA + 0.5 $\mu$ M pTPP 16.48 [-20.83, 53.79], Fig. 5B).

\section{Discussion}

Increased oxidative stress associated with some features of mitochondrial dysfunction was reported in skeletal muscle cells after the exposure to palmitate $[17,18]$. However, the effects of saturated fatty acids on mitochondrial respiration have not been well studied, particularly in undifferentiated skeletal muscle cells. The present study was designed to clarify the effect of palmitate on viability and mitochondrial respiratory function in differentiated and undifferentiated $\mathrm{C} 2 \mathrm{C} 12$ skeletal muscle cells and to test the ability of mitochondria-targeted antioxidants MitoQ and MitoTEMPOL to prevent palmitate-induced damage.

Palmitate appeared to be cytotoxic for myoblasts as well as myotubes in a dosedependent manner although myoblasts were much more sensitive. We have chosen a physiologically relevant concentration of palmitate $(100 \mu \mathrm{M})$ [35] to study further whether mtDNA integrity is affected in palmitate-treated cells. Mitochondrial DNA is one of the main targets for reactive oxygen species and its damage has been suggested as a marker of oxidative damage [38]. We found mtDNA damage after the treatment with palmitate in both myoblasts and myotubes using the QPCR method, which is able to detect a wide range of types of DNA lesions. Our observation is in accordance with another study [18] where mtDNA damage was detected in L6 myotubes exposed to palmitate using quantitative alkaline Southern blot analysis. Authors of this study postulated that mtDNA damage is a critical event leading to mitochondrial dysfunction with consequent increase in oxidative stress and finally to apoptosis [18]. In our experiments this was true only for myoblasts, as mtDNA damage after the exposure to palmitate was similar in myoblasts and myotubes but we detected mitochondrial dysfunction (discussed below) with increased hydrogen peroxide production (by about 50\%) and decreased viability (by about 35\%) only in myoblasts. It should be noted that the concentration of palmitate used in our study was lower than in most previous studies showing its deleterious effects in myotubes $[14,17,18]$.

Mitochondrial respiration after the treatment with palmitate was affected in different ways in myoblasts and myotubes. We observed decreased basal mitochondrial respiration, uncoupler-induced maximal respiration, spare respiratory capacity (SRC) and coupling efficiency in palmitate-treated myoblasts. A decrease in all parameters of mitochondrial respiration except the proton leak in palmitate-treated myoblasts could be explained by a reduction in mitochondrial mass as the activity of citrate synthase was significantly lower in palmitate-treated myoblasts. In myotubes, uncoupler-induced maximal respiration, SRC and the citrate synthase activity were not affected after the exposure to palmitate suggesting no change in mitochondrial respiratory capacity or mitochondrial density. Palmitate caused higher basal respiration because of an increased proton leak showing a slight uncoupling of mitochondrial respiration from oxidative phosphorylation, an effect already reported for long-chain fatty acids in skeletal muscle [39]. This effect could be, at least in part, mediated through the uncoupling protein-3 (UCP-3) which is specifically expressed in differentiated muscle cells [40] since we did not observe an increase in proton leak in undifferentiated myoblasts. Although the coupling efficiency, a portion of basal respiration used for ATP synthesis, was decreased after the treatment with palmitate; the rate of ATP turnover-linked respiration itself was not affected.

The presented results show that palmitate caused similar mtDNA damage in myoblasts and myotubes, which was associated with mitochondrial respiratory dysfunction and decreased viability only in myoblasts. We hypothesize that this higher resistance of myotubes to the effect of palmitate compared to myoblasts could be in part explained by their higher 
spare respiratory capacity (SRC, $75 \%$ of maximal OCR in myotubes vs. $53 \%$ in myoblasts) and therefore an increased ability to face the stress caused by palmitate. SRC is critical for survival and function of cells as it reflects the maximum ability of mitochondria to maintain energy production in response to an increase in energy demand, like in acute or chronic stress $[41,42]$.

Mitochondria-targeted antioxidants MitoQ and MitoTEMPOL used in this study have been previously shown to protect pancreatic $\beta$-cells against oxidative stress under glucolipotoxic conditions [24], inhibit peroxide-induced mitochondrial oxidative damage in endothelial cells [23] or prevent oxidative damage of mtDNA in myoblasts [27]. In our study, MitoTEMPOL prevented palmitate-induced mtDNA damage in C2C12 myoblasts and decreased production of hydrogen peroxide in non-treated cells confirming its antioxidant potential. In palmitate-treated myoblasts there was a trend towards a reduction of hydrogen peroxide emission but the difference did not reach statistical significance $(p=0.06)$. However, we did not observe a protective effect of MitoTEMPOL against palmitate-induced cell death or mitochondrial respiratory dysfunction. MitoQ had no significant effect on palmitate-induced mtDNA damage or decreased viability of myoblasts and caused even increased hydrogen peroxide production. Pro-oxidant properties of a higher dose of MitoQ $(1 \mu \mathrm{M})$ have been reported in certain conditions in isolated mitochondria $[43,44]$ while simultaneously noting its antioxidant potential. We tested even lower concentrations of antioxidants in toxicity assays, but they were also not able to prevent palmitate-induced cell death of myoblasts (Fig.4). When comparing with previous studies showing protective effects of antioxidants it should be taken into consideration that the effects will most probably depend on cell type, number of mitochondria in cells or the degree of reliance on oxidative phosphorylation.

Furthermore we found a strong inhibition of mitochondrial respiration after the treatment with both antioxidants. The functional impact of mitochondria-targeted antioxidants on cellular bioenergetics was shown recently for micromolar concentrations of MitoQ and MitoTEMPOL in mouse kidney mesangial cells [45]. We observed an inhibition of mitochondrial respiration also for $10 \mu \mathrm{M}$ concentration of propylTPP showing that the $\mathrm{TPP}^{+}$moiety itself could be the cause of this effect. This is again in agreement with the above mentioned work of Reily et al. [45] where the acute toxicity of TPP-conjugated compounds was tested and it was found that the $\mathrm{TPP}^{+}$moiety itself has a negative impact on cellular bioenergetics. Concentrations of targeted antioxidants should therefore be carefully selected and tested for potential bioenergetics effects and an "inactive" $\mathrm{TPP}^{+}$compound should always be included as a control.

We conclude that palmitate in physiologically relevant doses affected mainly myoblasts, which were more sensitive to its effects than differentiated myotubes, possibly due to a lower spare respiratory capacity. The results suggest that skeletal muscle progenitor cells may be the first target of the deleterious action of palmitate. Mitochondria-targeted antioxidants were unable to prevent palmitate-induced cell death in myoblasts although MitoTEMPOL prevented mtDNA damage. Both antioxidants as well as propylTPP markedly inhibited mitochondrial respiration in myoblasts. These results show that the effects of mitochondriatargeted antioxidants as well as $\mathrm{TPP}^{+}$compounds on cellular bioenergetics need to be further studied and taken into account when used in cell culture studies or for therapeutic purposes.

\section{Disclosure Statement}

The authors confirm that there are no conflicts of interest.

\section{Acknowledgements}

This work was supported by the research project of Grant Agency of Charles University GAUK 661912, 266704/SVV/2013 and PRVOUK-P31. 


\section{References}

1 Reaven GM, Hollenbeck C, Jeng CY, Wu MS, Chen YD: Measurement of plasma glucose, free fatty acid, lactate, and insulin for $24 \mathrm{~h}$ in patients with NIDDM. Diabetes 1988;37:1020-1024.

- Groop LC, Saloranta C, Shank M, Bonadonna RC, Ferrannini E, DeFronzo RA: The role of free fatty acid metabolism in the pathogenesis of insulin resistance in obesity and noninsulin-dependent diabetes mellitus. J Clin Endocrinol Metab 1991;72:96-107.

3 Karpe F, Dickmann JR, Frayn KN: Fatty acids, obesity, and insulin resistance: time for a reevaluation. Diabetes 2011;60:2441-2449.

- 4 Belfort R, Mandarino L, Kashyap S, Wirfel K, Pratipanawatr T, Berria R, DeFronzo RA, Cusi K: Dose-response effect of elevated plasma free fatty acid on insulin signaling. Diabetes 2005;54:1640-1648.

-5 Lee JS, Pinnamaneni SK, Eo SJ, Cho IH, Pyo JH, Kim CK, Sinclair AJ, Febbraio MA, Watt MJ: Saturated, but not $n-6$ polyunsaturated, fatty acids induce insulin resistance: role of intramuscular accumulation of lipid metabolites. J Appl Physiol 2006;100:1467-1474.

-6 Hoeks J, van Herpen NA, Mensink M, Moonen-Kornips E, van Beurden D, Hesselink MKC, Schrauwen P: Prolonged Fasting Identifies Skeletal Muscle Mitochondrial Dysfunction as Consequence Rather Than Cause of Human Insulin Resistance. Diabetes 2010;59:2117-2125.

7 Coll T, Eyre E, Rodríguez-Calvo R, Palomer X, Sánchez RM, Merlos M, Laguna JC, Vázquez-Carrera M: Oleate reverses palmitate-induced insulin resistance and inflammation in skeletal muscle cells. J Biol Chem 2008;283:11107-11116.

-8 Pimenta AS, Gaidhu MP, Habib S, So M, Fediuc S, Mirpourian M, Musheev M, Curi R, Ceddia RB: Prolonged exposure to palmitate impairs fatty acid oxidation despite activation of AMP-activated protein kinase in skeletal muscle cells. J Cell Physiol 2008;217:478-485.

-9 Hirabara SM, Curi R, Maechler P: Saturated fatty acid-induced insulin resistance is associated with mitochondrial dysfunction in skeletal muscle cells. J Cell Physiol 2010;222:187-194.

10 Roden M, Price TB, Perseghin G, Petersen KF, Rothman DL, Cline GW, Shulman GI: Mechanism of free fatty acid-induced insulin resistance in humans. J Clin Invest 1996;97:2859-2865.

11 Brownlee M: The pathobiology of diabetic complications: a unifying mechanism. Diabetes 2005;54:16151625.

12 Bonnard C, Durand A, Peyrol S, Chanseaume E, Chauvin M-A, Morio B, Vidal H, Rieusset J: Mitochondrial dysfunction results from oxidative stress in the skeletal muscle of diet-induced insulin-resistant mice. J Clin Invest 2008;118:789-800.

13 Anderson EJ, Lustig ME, Boyle KE, Woodlief TL, Kane DA, Lin C et al.: Mitochondrial H2O2 emission and cellular redox state link excess fat intake to insulin resistance in both rodents and humans. J Clin Invest 2009;119:573-581.

14 Rachek LI, Musiyenko SI, LeDoux SP, Wilson GL: Palmitate induced mitochondrial deoxyribonucleic acid damage and apoptosis in L6 rat skeletal muscle cells. Endocrinology 2007;148:293-299.

-15 Duval C, Cámara Y, Hondares E, Sibille B, Villarroya F: Overexpression of mitochondrial uncoupling protein-3 does not decrease production of the reactive oxygen species, elevated by palmitate in skeletal muscle cells. FEBS Lett 2007;581:955-961.

16 Lambertucci RH, Hirabara SM, Silveira LDR, Levada-Pires AC, Curi R, Pithon-Curi TC: Palmitate increases superoxide production through mitochondrial electron transport chain and NADPH oxidase activity in skeletal muscle cells. J Cell Physiol 2008;216:796-804.

17 Barbosa MR, Sampaio IH, Teodoro BG, Sousa TA, Zoppi CC, Queiroz AL et al.: Hydrogen peroxide production regulates the mitochondrial function in insulin resistant muscle cells: effect of catalase overexpression. Biochim Biophys Acta 2013;1832:1591-1604.

-18 Yuzefovych LV, Solodushko VA, Wilson GL, Rachek LI: Protection from palmitate-induced mitochondrial DNA damage prevents from mitochondrial oxidative stress, mitochondrial dysfunction, apoptosis, and impaired insulin signaling in rat L6 skeletal muscle cells. Endocrinology 2012;153:92-100.

19 Jauslin ML, Meier T, Smith RA, Murphy MP: Mitochondria-targeted antioxidants protect Friedreich Ataxia fibroblasts from endogenous oxidative stress more effectively than untargeted antioxidants. FASEB J 2003;17:1972-1974.

20 Green K, Brand MD, Murphy MP: Prevention of mitochondrial oxidative damage as a therapeutic strategy in diabetes. Diabetes 2004;53 Suppl 1:S110-118. 


\section{Cellular Physiology $\quad$ Cell Physiol Biochem 2014;33:1439-1451 and Biochemistry \\ Patková/Anděl/Trnka: Palmitate and Mitochondrial Dysfunction}

21 Murphy MP, Smith RA: Targeting antioxidants to mitochondria by conjugation to lipophilic cations. Annu Rev Pharmacol Toxicol 2007;47:629-656.

22 Smith RA, Porteous CM, Coulter CV, Murphy MP: Selective targeting of an antioxidant to mitochondria. Eur J Bioch 1999;263:709-716.

-23 Dhanasekaran A, Kotamraju S, Kalivendi SV, Matsunaga T, Shang T, Keszler A, Joseph J, Kalyanaraman B: Supplementation of endothelial cells with mitochondria-targeted antioxidants inhibit peroxide-induced mitochondrial iron uptake, oxidative damage, and apoptosis. J Biol Chem 2004;279:37575-37587.

24 Lim S, Rashid MA, Jang M, Kim Y, Won H, Lee J, Woo J, Kim YS, Murphy MP, Ali L, Ha J, Kim SS: Mitochondriatargeted antioxidants protect pancreatic $\beta$-cells against oxidative stress and improve insulin secretion in glucotoxicity and glucolipotoxicity. Cell Physiol Biochem 2011;28:873-886.

25 Adlam VJ, Harrison JC, Porteous CM, James AM, Smith RA, Murphy MP, Sammut IA: Targeting an antioxidant to mitochondria decreases cardiac ischemia-reperfusion injury. FASEB J 2005;19:1088-1095.

26 Smith RA, Murphy MP: Animal and human studies with the mitochondria-targeted antioxidant MitoQ. Ann NY Acad Sci 2010;1201:96-103.

27 Trnka J, Blaikie FH, Logan A, Smith RA, Murphy MP: Antioxidant properties of MitoTEMPOL and its hydroxylamine. Free Radical Res 2009;43:4-12.

28 Cousin SP, Hügl SR, Wrede CE, Kajio H, Myers MG, Rhodes CJ: Free fatty acid-induced inhibition of glucose and insulin-like growth factor I-induced deoxyribonucleic acid synthesis in the pancreatic beta-cell line INS-1. Endocrinology 2001;142:229-240.

29 Spector AA: Fatty acid binding to plasma albumin. J Lipid Res 1975;16:165-179.

-30 Smith RA, Kelso GF, James AM, Murphy MP: Targeting coenzyme Q derivatives to mitochondria. Meth Enzymol 2004;382:45-67.

- 31 Santos JH, Meyer JN, Mandavilli BS, Van Houten B: Quantitative PCR-based measurement of nuclear and mitochondrial DNA damage and repair in mammalian cells. Methods Mol Biol 2006;314:183-199.

- 32 Ayala-Torres S, Chen Y, Svoboda T, Rosenblatt J, Van Houten B: Analysis of gene-specific DNA damage and repair using quantitative polymerase chain reaction. Methods 2000;22:135-147.

33 Brand MD, Nicholls DG: Assessing mitochondrial dysfunction in cells. Biochem J 2011;435:297-312.

34 Ainscow EK, Brand MD: Top-down control analysis of ATP turnover, glycolysis and oxidative phosphorylation in rat hepatocytes. Eur J Bioch 1999;263:671-685.

- 35 Kien CL, Bunn JY, Poynter ME, Stevens R, Bain J, Ikayeva O, Fukagawa NK, Champagne CM, Crain KI, Koves TR, Muoio DM: A lipidomics analysis of the relationship between dietary fatty acid composition and insulin sensitivity in young adults. Diabetes 2013;62:1054-1063.

- 36 Kelso GF, Porteous CM, Coulter CV, Hughes G, Porteous WK, Ledgerwood EC, Smith RA, Murphy MP: Selective targeting of a redox-active ubiquinone to mitochondria within cells: antioxidant and antiapoptotic properties. J Biol Chem 2001;276:4588-4596.

-37 Marthandan S, Murphy MP, Billett E, Barnett Y: An investigation of the effects of MitoQ on human peripheral mononuclear cells. Free Radical Res 2011;45:351-358.

- 38 Yakes FM, Van Houten B: Mitochondrial DNA damage is more extensive and persists longer than nuclear DNA damage in human cells following oxidative stress. P Natl Acad Sci USA 1997;94:514-519.

-39 Hirabara SM, Silveira LR, Alberici LC, Leandro CVG, Lambertucci RH, Polimeno GC, Boaventura MFC, Procopio J, Vercesi AE, Curi R: Acute effect of fatty acids on metabolism and mitochondrial coupling in skeletal muscle. Biochim Biophys Acta 2006;1757:57-66.

-40 Cámara Y, Duval C, Sibille B, Villarroya F: Activation of mitochondrial-driven apoptosis in skeletal muscle cells is not mediated by reactive oxygen species production. Int J Biochem Cell B 2007;39:146-160.

41 Dranka BP, Hill BG, Darley-Usmar VM: Mitochondrial reserve capacity in endothelial cells: The impact of nitric oxide and reactive oxygen species. Free Radic Biol Med 2010;48:905-914.

-42 Sansbury BE, Jones SP, Riggs DW, Darley-Usmar VM, Hill BG: Bioenergetic function in cardiovascular cells: the importance of the reserve capacity and its biological regulation. Chem Biol Interact 2011;191:288-295.

-43 James AM, Cochemé HM, Smith RA, Murphy MP: Interactions of mitochondria-targeted and untargeted ubiquinones with the mitochondrial respiratory chain and reactive oxygen species. Implications for the use of exogenous ubiquinones as therapies and experimental tools. J Biol Chem 2005;280:21295-21312.

-44 O'Malley Y, Fink BD, Ross NC, Prisinzano TE, Sivitz WI: Reactive oxygen and targeted antioxidant administration in endothelial cell mitochondria. J Biol Chem 2006;281:39766-39775.

45 Reily C, Mitchell T, Chacko BK, Benavides G, Murphy MP, Darley-Usmar V: Mitochondrially targeted compounds and their impact on cellular bioenergetics. Redox Biol 2013;1:86-93. 